\title{
Nanoparticles Actively Fragment Armored Droplets
}

\author{
François Sicard, ${ }^{* \dagger} \odot$ Jhoan Toro-Mendoza, $\stackrel{\ddagger}{\dagger}$ and Alberto Striolo ${ }^{\S \odot}$ \\ ${ }^{\dagger}$ Department of Chemistry, King's College London, SE1 1DB London, United Kingdom \\ ${ }^{\ddagger}$ Centro de Estudios Interdisciplinarios de la Fisica, Instituto Venezolano de Investigaciones Cientificas, Caracas 1020A, Venezuela \\ ${ }^{\S}$ Department of Chemical Engineering, University College London, WC1E 7JE London, United Kingdom
}

\section{Supporting Information}

ABSTRACT: Understanding the complexity of fragmentation processes is essential for regulating intercellular communication in mechanistic biology and developing bottom-up approaches in a large range of multiphase flow processes. In this context, self-fragmentation proceeds without any external mechanical energy input, allowing one to create efficiently micro- and nanodroplets. Here we examine self-fragmentation in emulsion nanodroplets stabilized by solid particles with different surface features. Mesoscopic modeling and accelerated dynamics simulations allow us to overcome the limitations of atomistic simulations and offer detailed insight into the interplay between the evolution of the droplet shape and the particle finite-size effects at the interface. We show that finite-size nanoparticles play an active role in the necking breakup, behaving like nanoscale razors, and affect strongly the thermodynamic properties of the system. The role played by the particles during self-fragmentation might be of relevance to multifunctional biomaterial design and tuning of signaling pathways in mechanistic biology.

KEYWORDS: self-fragmentation, emulsion droplet, nanoparticle, free-energy, accelerated dynamics simulation

1 mulsion droplets are metastable dispersions composed of two immiscible fluids such as water and oil. ${ }^{1,2}$ The associated surface tension forces them into a spherical shape to minimize the free-energy. To decrease the latter and stabilize the emulsion droplets, a surface-active agent can be added. ${ }^{3,4}$ In particular Pickering emulsions are stabilized by the incorporation of particles. ${ }^{3,5}$ Emulsion droplets can serve as ideal compartments for reactions catalyzed by nanoparticles (NPs) attached at the oil-water interfaces ${ }^{6}$ and can be used as drug-delivery vehicles, ${ }^{7}$ sensors, ${ }^{8}$ and templates for the fabrication of advanced functional materials. ${ }^{9-11}$ The characteristics of Pickering emulsions pose a number of intriguing fundamental physical questions, including a thorough understanding of the perennial lack of detail about how particles arrange at the liquid/liquid interface. Other not completely answered questions include particle effects on interfacial tension, ${ }^{12}$ layering, ${ }^{13}$ buckling, ${ }^{14}$ and droplet bridging. ${ }^{15}$ Interestingly, emulsion droplets show some relevant characteristics and qualities of living systems that could make them proxies for artificial life. They provide an experimental framework for synthetic biology that is different from other protocell model systems such as vesicles offering distinct advantages. ${ }^{17-19}$ For instance, the apparent similarity in surface properties between inorganic NPs and globular proteins ${ }^{20}$ and the fluid dynamical properties of droplets could be combined with different chemistries to target applications and exploration of biological scenarios not easily achievable with other supramolecular platforms, ${ }^{21}$ including the mechanical biology of enveloped viruses such as the rapidly spreading Zika virus. $^{22-24}$

In the presence of colloidal particles, the stability of emulsions against fragmentation under ultrasonication ${ }^{25-27}$ or shear stress ${ }^{28,29}$ has been a subject of strong interest either at the molecular or mesoscopic level. However, despite the vast interest in particle-laden interfaces, less is known about the self-fragmentation mechanism, that is, fragmentation without any external mechanical energy input. We refer to selffragmentation as to any mechanism based on thermally activated surface or local fluid current fluctuations. Such mechanisms can be described as random fluctuations, which could result in a deformation along a preferentially aligned direction. We describe below how such a process could lead to droplet rupture. For instance, Tcholakova et $_{\text {al. }}{ }^{30}$ studied a selfemulsification process via cooling-heating cycles causing a repeating breakup of droplets to higher-energy states. However, such an approach relies on small changes in

Received: June 7, 2019

Accepted: August 1, 2019

Published: August 1, 2019 
temperature affecting the spontaneous curvature due to surfactant thermally activated modification. We are here interested in self-fragmentation at constant temperature, as it is observed in the proliferation of living cells. ${ }^{17-19,21}$

Due to their inherent limited resolution, direct access to local observables, such as the particles' three-phase contact angle distribution, the measure of decrease in interfacial tension with the decrease in droplet size, as characterized by the Tolman length, ${ }^{31}$ or the presence of particles remain out of reach. These pieces of information can be accessed by numerical simulations. All-atom molecular dynamics (MD) simulations have become a widely employed computational technique. However, all-atom MD simulations are computationally expensive. Moreover, most phenomena of interest can take place on time scales that are orders of magnitude longer than those accessible via all-atom MD. Mesoscopic simulations, in which the structural unit is a coarse-grained representation of a large number of molecules, and enhanced sampling techniques allow us to overcome these limitations. Coarse-grained approaches offer the possibility of answering fundamental questions responsible for the collective behavior of particles anchored at an interface. ${ }^{32}$

We employ here dissipative particle dynamics (DPD) as a mesoscopic simulation method ${ }^{33}$ along with accelerated dynamics simulations ${ }^{34}$ to study accurately the free-energy landscapes and the necking breakup mechanisms associated with thermally assisted self-fragmentation of model water nanodroplets coated with spherical NPs and immersed in an organic solvent. The scaled temperature in the DPD framework is equivalent to $298.73 \mathrm{~K}$. The procedure and parametrization details are described in the Supporting Information (SI). The particles are of two types: Janus (particles whose surface shows two distinct wetting properties) and homogeneous (particles with one surface characteristic). They are chosen so that the initial three-phase contact angles $\left(\approx 90^{\circ}\right)$ result in maximum adsorption energy. ${ }^{35}$ To study the thermodynamic properties of the self-fragmentation mechanism and the role played by the finite-size particles, we combined several enhanced sampling frameworks, including metadynamics (metaD), ${ }^{36}$ umbrella sampling (US), ${ }^{37}$ and adiabatic biased molecular dynamics (ABMD). ${ }^{38,39}$ To take into account the inherent fluid nature of the system, we designed a dynamic collective variable (CV) allowing to bias unequivocally the dynamics of the system based on dynamical labeling of the fluid molecules composing the emulsion droplet as well as the mother and the daughter droplets resulting from self-fragmentation (see Methods). We consider throughout this study the same number of fluid molecules constituting the bare or armored emulsion nanodroplets and the same NP density on the armored nanodroplets.

\section{RESULTS AND DISCUSSION}

In Figure 1 we show representative snapshots obtained in the accelerated dynamics simulations for the bare emulsion nanodroplet (panel a) and a system stabilized with either Janus (panel b) or homogeneous (panel c) NPs. Starting with the spherical bare droplet (configuration $E_{1}$ in Figure $1 \mathrm{a}$ ), the system fragments with the formation of a liquid bridge whose shape approximately describes an evolving parabola $\left(E_{2}-E_{6}\right)$. The curvature of the liquid bridge increases continuously along the fragmentation process until mother and daughter nanodroplets separate, resulting in two distinct emulsion nano- (a)

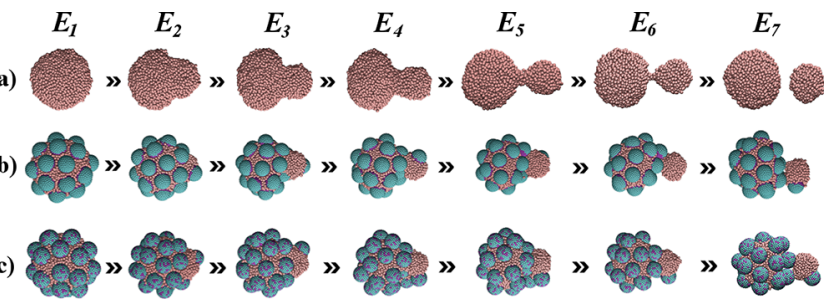

Figure 1. Sequence of simulation snapshots representing the thermally assisted self-fragmentation processes of water in oil nanodroplets in the bare configuration (a) or armored with 24 spherical Janus (b) and homogeneous (c) NPs. The bare and armored nanodroplets are constituted of the same number of water beads, $N_{\mathrm{W}} \approx 3500$. The same $\mathrm{NP}$ surface coverage $\phi \approx 0.9$, as defined in ref 16 , is considered on the armored nanodroplets. The NPs simulated have the same volume, $4 / 3 \pi a_{0}^{3}$, where $a_{0} \approx 1.5$ $\mathrm{nm}$. This yields the radius of gyration, $R_{\mathrm{GYR}}=3.764 \pm 0.004 \mathrm{~nm}$, and $R_{\mathrm{GYR}}=3.953 \pm \mathbf{0 . 0 0 8} \mathrm{nm}$ for bare and armored nanodroplets, respectively. Cyan and purple spheres represent polar and apolar beads. Pink spheres represent water beads. The oil beads surrounding the droplets are not shown for clarity. The number of contacts, $N_{\mathrm{C}}$ (see Methods), decreases from left to right, with $E_{1}$ corresponding to the original configuration and $E_{7}$ to the final configuration obtained within the accelerated dynamics framework.

droplets with radius of gyration $R_{\mathrm{GYR}}^{(\mathrm{m})}=3.521 \pm 0.004 \mathrm{~nm}$ and $R_{\mathrm{GYR}}^{(\mathrm{d})}=2.188 \pm 0.007 \mathrm{~nm}$, respectively $\left(E_{7}\right)$.

The fragmentation process is fundamentally different when the emulsion droplet is stabilized with either Janus or homogeneous NPs ( $c f$. Figure 1b,c). As the particles remain strongly adsorbed at the interface during fragmentation, forming a close-packed monolayer, the emulsion nanodroplet fragments forming first a liquid bridge similar to the one observed in the bare droplet (configuration $E_{2}$ in Figure 1).

Progressively, the particles located at the fragmentation edge actively control the process. For instance, they decrease the number of contacts between mother and daughter droplets. As shown in Figure 2, these edging NPs control the shape of the bridge between the 2 droplets, behaving like nanoscale razors, and transform the liquid bridge in bridging filaments. Finally, the bridging filaments vanish and mother and daughter emulsion droplets separate.
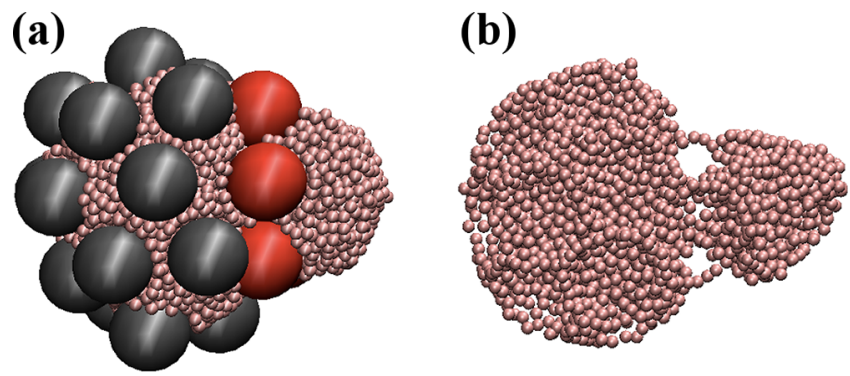

Figure 2. Simulation snapshot obtained during the thermally assisted self-fragmentation process of the emulsion droplet armored with Janus NPs. (a) The edging NPs separating mother and daughter droplets are colored in red. The particles which are not involved in the fragmentation process are colored in gray. (b) Illustration of the filament bridge formed in the necking breakup mechanism when the particles in panel (a) are not shown. Pink spheres represent water beads. The oil beads are not shown for clarity. 

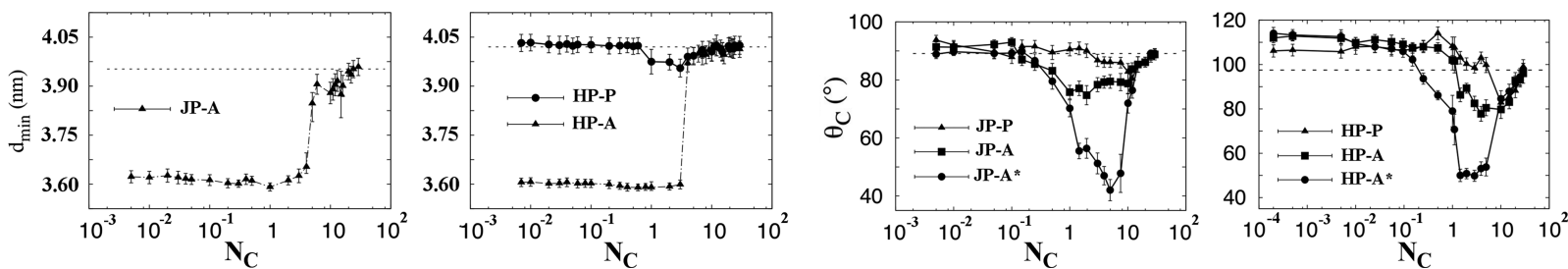

Figure 3. Evolution of the representative minimal distances $d_{\min }$ (left panels) measured between the edging NPs and the representative threephase contact angles $\theta_{\mathrm{C}}$ (right panels) of Janus (JP) and homogeneous (HP) edging NPs as a function of the number of contacts $N_{\mathrm{C}}$ between mother and daughter droplets (see Methods). The letters $\mathbf{P}$ and $\mathbf{A}$ are used to differentiate the NPs which play a passive and an active role in the breakup mechanism, respectively. The statistical errors are estimated as 1 standard deviation (SD) from the mean as obtained for equilibrated trajectories. The values $d_{\min }$ and $\theta_{\mathrm{C}}$ of a representative NP far from the fragmentation area are plotted in dashed line for reference.

When the armored nanodroplet is coated with Janus NPs (Figure 1b), their hydrophobic regions interact with the daughter droplet, pushing it further away from the mother droplet. The final configuration is fundamentally different when the water nanodroplet is stabilized with homogeneous NPs (Figure 1c). Their specific feature allows the edging homogeneous NPs to adsorb both at the mother and daughter droplet interfaces once they are separated, resulting in bridging. ${ }^{39}$ As the distance between mother and daughter droplets is not sufficiently large, the fragmented emulsion droplets can coalesce.

The breakup mechanism just discussed is quantitatively investigated in Figure 3 (left panels), where we show the evolution of a representative set of minimal distances, $d_{\min }$, measured between the edging NPs separating mother and daughter nanodroplets as a function of the number of contacts, $N_{\mathrm{C}}$, between the water molecules in each droplets. The initial distributions of the minimal distances can be described with Gaussian distributions for both Janus (J) and homogeneous $(\mathrm{H})$ NPs. The values of the respective means, $\mu^{\mathrm{J}}$ and $\mu^{\mathrm{H}}$, and standard deviations, $\sigma^{\mathrm{J}}$ and $\sigma^{\mathrm{H}}$, differ due to the NP features. We obtain $\mu^{\mathrm{J}}=3.96 \mathrm{~nm}$ and $\mu^{\mathrm{H}}=4.02 \mathrm{~nm}$ and $\sigma^{\mathrm{J}}=0.03 \mathrm{~nm}$ and $\sigma^{\mathrm{H}}=0.02 \mathrm{~nm}$. As the number of contacts $N_{\mathrm{C}}$ between mother and daughter droplets coated with Janus NPs decreases, the minimal distances between the edging NPs show two distinct regimes separated with a transition at $N_{\mathrm{C}}^{*} \approx$ 5. When $N_{\mathrm{C}}>N_{\mathrm{C}}^{*}, d_{\min }$ decreases slowly as the liquid bridge forms, similar to the one observed in the bare droplet. When $N_{\mathrm{C}} \approx N_{\mathrm{C}}^{*}, d_{\min }$ shows a significant jump which is characteristic of the transition from the liquid bridge to the bridging filaments shown in Figure 2. When $N_{\mathrm{C}}<N_{\mathrm{C}}^{*}, d_{\min }$ decreases continuously until the bridging filaments vanish at $N_{\mathrm{C}}^{\dagger} \approx 1$ and mother and daughter droplets separate. For $N_{\mathrm{C}}<N_{\mathrm{C}}^{\dagger}, d_{\text {min }}$ increases slightly until a plateau is reached, which is characteristic of the local rearrangement of the edging NPs near the fragmentation area. The evolution of the system is qualitatively similar when homogeneous NPs are present, albeit some subtle differences emerge due to the NP features. As shown in Figure 3, two distinct behaviors are observed. Unlike Janus edging NPs, certain homogeneous NPs do not present a distance $d_{\min }$ showing a significant jump when $N_{\mathrm{C}}=N_{\mathrm{C}}^{*}$, but follow a relatively small and continuous decrease for $N_{\mathrm{C}}^{\dagger}<N_{\mathrm{C}}$. The rest of the evolution is similar to the one observed for Janus NPs, with the continuous increase of $d_{\min }$ to the plateau characteristic of the local equilibrium rearrangement of the edging NPs.

This analysis can be completed by quantifying the evolution of the three-phase contact angles, $\theta_{\mathrm{C}}$, of the edging NPs, as shown in Figure 3 (right panels). The initial distribution can be described with Gaussian distributions for both Janus $(\mathrm{J})$ and homogeneous $(\mathrm{H}) \mathrm{NPs}$, with respective means and standard deviations $\mu^{\mathrm{J}}=89.1^{\circ}$ and $\mu^{\mathrm{H}}=97.5^{\circ}$ and $\sigma^{\mathrm{J}}=1.6^{\circ}$ and $\sigma^{\mathrm{H}}=$ $3.5^{\circ}$. As the number of contacts $N_{C}$ between mother and daughter droplets coated either with Janus or homogeneous NPs decreases, two distinct behaviors emerged, which could discriminate the active or passive role played by the edging NPs in the self-fragmentation process. When $N_{C}>N_{C}^{*}$, the three-phase contact angle decreases slowly as the liquid bridge forms. When $N_{\mathrm{C}}^{\dagger}<N_{\mathrm{C}}<N_{\mathrm{C}}^{*}$, we observe two different evolutions of the contact angle. Some NPs show a continuous and significant decrease of $\theta_{\mathrm{C}}$, with a decrease of $\approx 20 \%$ (index $A$ in Figure 3 ) and $\approx 50 \%$ (index $A^{*}$ in Figure 3 ). This behavior is representative of the active role played by some edging NPs which behave like nanoscale razors. Eventually, the bridging filaments vanish when $N_{\mathrm{C}}=N_{\mathrm{C}}^{\dagger}$ and mother and daughter droplets separate for $N_{\mathrm{C}}^{\dagger}<N_{\mathrm{C}}$. This step is characterized by a significant increase of the contact angle until it reaches a plateau associated with the local rearrangement of the edging NPs resulting in higher values of the contact angles near the fragmentation area. In contrast, some edging NPs show an increase of their contact angle when $N_{\mathrm{C}}<$ $N_{C}^{*}$, until it reaches the plateau, characteristic of their passive role during the breakup mechanism (index P in Figure 3).

Building on accelerated dynamics frameworks, ${ }^{34}$ we assessed the free-energy of self-fragmentation for bare and armored nanodroplets. Considering first the bare droplet, we obtained $\Delta F_{\mathrm{B}}=445 \pm 3 \mathrm{kcal} / \mathrm{mol}$. This value can be compared with the expression of the Gibbs free-energy, ${ }^{40} \Delta F=\gamma \Delta A$, where $\gamma$ and $\Delta A$ are the liquid-liquid interfacial tension and the change in interfacial area, respectively. Taking into account the effect of the curvature of the emulsion nanodroplet, ${ }^{31,41}$ one must consider $\gamma=\gamma_{0} /\left(1+2 \delta / R_{\mathrm{S}}\right)$, where $\gamma_{0}, R_{\mathrm{S}}$, and $\delta$ are the planar interfacial tension, the radius of the surface under tension, and the Tolman length, respectively. Given the interfacial tension for a planar decane/water interface, ${ }^{42} \gamma_{0}=51.7 \mathrm{mN} \cdot \mathrm{m}^{-1}$, we obtained $\Delta F_{0}=467 \pm 5 \mathrm{kcal} / \mathrm{mol}$, which yields the value of the Tolman length $\delta \approx 10^{-8} \mathrm{~cm}$, in agreement with the original paper of Tolman. ${ }^{31}$ Noticeably, this latter result was directly obtained from our simulations and not as a condition imposed where a definition of surface density profiles is needed in atomistic descriptions.

In Figure 4, we compared this result with the free-energy of self-fragmentation for the armored nanodroplets, $\Delta F_{\mathrm{J}}$ and $\Delta F_{\mathrm{H}}$, respectively, also obtained within the accelerated dynamics framework. As we could expect from the quantitative analysis above, which highlighted the active role played by the finitesize NPs in the breakup mechanism, we measured $\Delta F_{\mathrm{J}}=316 \pm$ $7 \mathrm{kcal} / \mathrm{mol}$ and $\Delta F_{\mathrm{H}}=227 \pm 3 \mathrm{kcal} / \mathrm{mol}$, significantly lower 


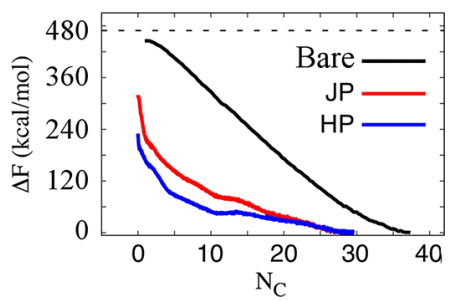

Figure 4. Free-energy profiles associated with thermally assisted self-fragmentation of bare and armored droplets as a function of the number of contacts $N_{\mathrm{C}}$ between mother and daughter droplets (see Methods). The Gibbs free-energy calculated without the Tolman correction is shown as a dashed horizontal line. The positions of the global minima of the bare and armored droplets along $N_{\mathrm{C}}$ differ due to the presence of the particles.

than the free-energy of self-fragmentation measured for the bare nanodroplet. Furthermore, particle adsorption at the two interfaces when homogeneous NPs are present must be taken into account to estimate the fragmentation free-energy. To do so, we measured the three-phase contact angle of the edging NPs and defined the final fragmentation stage as the one where the edging NPs remain adsorbed at a single interface. In Figure 5 , we show the evolution of the representative three-phase

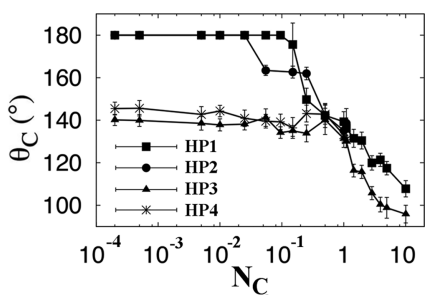

Figure 5. Representative three-phase contact angles $\theta_{C}$ of the homogeneous (HP) edging NPs with respect to the daughter droplet as the fragmentation process progresses from stage $\mathrm{E} 4$ to E7 as a function of the continuous number of contacts $N_{C}$ between mother and daughter droplets (see Methods). The statistical errors are estimated as 1 SD from the mean for equilibrated trajectories.

contact angles of the edging NPs with respect to the daughter droplet as the fragmentation process progresses from stage $E_{4}$ to $E_{7}$, as shown in Figure 1 . As the edging particles $\mathrm{HP}_{1}$ and $\mathrm{HP}_{2}$ are fully desorbed when the number of contacts $N_{\mathrm{C}} \approx$ $10^{-2}$, with a three-phase contact angle $\theta_{\mathrm{C}}=180^{\circ}$, two particles, $\mathrm{HP}_{3}$ and $\mathrm{HP}_{4}$, have a contact angle $\theta_{\mathrm{C}}<180^{\circ}$ characteristic of particles partially adsorbed at the daughter interface. In the Pieranski-Binks approach, ${ }^{35,43}$ the change in energy accompanying the desorption of a spherical particle from the interface to either bulk phase can be approximated by $\Delta E=$ $\pi r^{2} \gamma_{0}(1 \pm \cos \theta)^{2}$, where $r$ is the particle radius and the plus (minus) sign refers to desorption into oil (water). Even if this expression assumes that the oil-water interface remains planar, it yields a rough approximation of the energy at play. Considering the contact angles given in Figure 5, we obtain a correction factor $\Delta \Delta F_{\mathrm{H}} \approx 5 \mathrm{kcal} / \mathrm{mol}$.

Interestingly, the difference in chemistry of the spherical NPs had some important impact in the reduction of the freeenergy barrier. Unlike Janus NPs, which present a preferred orientation at the liquid-liquid interface resulting in restricted rotational mobility, homogeneous NPs are characterized by larger rotational freedom. This provides the homogeneous NPs with the capability to share the interfacial area delimiting mother and daughter nanodroplets during the fragmentation process. In contrast, when Janus NPs are present, the main energy consumption comes from the daughter droplet trying to maintain the orientation of the edging NPs, thus increasing the free-energy of self-fragmentation.

\section{CONCLUSIONS}

The physical insights discussed here provide a deeper understanding of the organization of finite-size NPs at fluid interfaces and allow us to decipher the active or passive role played by such particles in the self-fragmentation process. This information could be useful for a variety of applications including multifunctional biomaterial design and tuning of signaling pathways in mechanistic biology, such as membranebound and membrane-less organelles during apoptosis. ${ }^{4-46}$ Apoptosis is a form of programmed cell death that is a highly regulated and controlled biological process, ${ }^{47}$ which can alter organelle structure and function. ${ }^{44}$ Its original role is to kill infected, abnormal, or otherwise undesired cells. There is a long list of diseases associated with altered cell survival. Increased apoptosis is characteristic of AIDS and neurodegenerative diseases such as Alzheimer's and Parkinson's. Decreased or inhibited apoptosis, on the other hand, is a feature of many malignancies, autoimmune disorders, and some viral infections. ${ }^{48}$ For instance, increasing evidence suggests that cell-derived extracellular vesicles (EV) produced during apoptosis have important immune regulatory roles relevant across different disease settings. ${ }^{49}$ The formation of EVs during apoptosis could be a key mechanism of immune modulation. With most cancer treatments focusing on inducing apoptosis in tumor cells, ${ }^{50}$ it becomes important to understand selective ways to influence cell differentiation and death. Nanoscale chemistry and topography could act synergistically for better understanding hidden mechanisms of nanomaterialinduced cell behaviors. From this perspective, emulsion droplets can promote open thinking about how nonliving matter might self-organize into evolving matter that adapts over time to a changing environment. However, directly sampling experimentally the role played by NPs remains challenging. Despite these difficulties, Pak et al. ${ }^{51}$ recently suggested it is possible to quantify the active implication of NPs on emulsification processes. This and other experimental setups could shed light on the richness of the selffragmentation process discussed here and advance the study of much more complex phenomena associated with various physicochemical conditions.

The extensive simulations discussed above allowed us to unravel thermally assisted self-fragmention of armored nanodroplet along with the mechanisms at play in the necking breakup process. We showed that finite-size NPs can play an active role, behaving like nanoscale razors, during the evolution of the droplet shape and affect strongly the stability of the system, resulting in significant reduction of the fragmentation free-energy ranging between 150 and $250 \mathrm{kcal} / \mathrm{mol}$, which is equivalent to the energy released by 20-30 ATP molecules. ${ }^{52}$ The relatively small size of the mother droplet considered in this work $(\approx 10 \mathrm{~nm}$ in diameter) comes from computational limitations due to dynamical labeling of the fluid molecules. Nevertheless, the DPD framework, along with continuously increasing computational power, would allow extending these results to a range of bioinspired liquid-liquid systems where the adsorption of the particles does not lead to a significant deformation of the interface. This is a valid approximation for 
particles and emulsion droplets ranging from nanometer to micrometer size. ${ }^{32}$ The results presented here also suggest that the effect of particle adsorption energy and particle density coverage affects particles' ability to fragment the emulsion droplet. For instance, increasing the initial three-phase contact angles and/or the particles' surface density would affect the formation of filaments, eventually yielding the formation of the liquid bridge observed in the fragmentation of the bare emulsion droplet. These observations might be of relevance for the control and/or tuning of the fragmentation of cell-derived extracellular vesicles, which have important immune regulatory roles, ${ }^{46}$ and bacterial membrane vesicles that affect diverse biological processes, including virulence, phage infection, and cell-to-cell communication. ${ }^{53}$ They might also enhance the design of nanoscale platforms in synthetic biology to modify the molecular workings of enveloped viruses such as the rapidly spreading Zika virus whose recent outbreak has been linked to microcephaly and Guillain-Barré syndrome. ${ }^{22-24}$

\section{METHODS}

Dynamical Coordination Number. To take into account the inherent fluid nature of the system, that is, the absence of covalent interactions between the molecules of the fluids, we designed a dynamic collective variable (CV) which allowed us to bias unequivocally the dynamics of the system based on dynamical labeling the fluid molecules composing mother and daughter droplets resulting from the self-fragmentation process. In this optic, we considered the MULTICOLVAR module of the plugin for free-energy calculation, PLUMED, version 2.3. ${ }^{54}$

- We first arbitrarily aligned the principal axis of deformation of the system with the Z-Cartesian axis of the DPD simulation box.

- We settled the fragmentation center as our reference position in the Cartesian space using a virtual atom in a fixed position with the FIXEDATOM function available in PLUMED. ${ }^{54}$

- We used the function ZDISTANCES to calculate the $Z$ components of the vectors connecting the fluid molecules constituting the droplet and the fragmentation center.

- We filtered the distribution of distances obtained with the ZDISTANCES function to dynamically discriminate the molecules located on the right (left) side of the fragmentation center. To do so, we used the function MFILTER MORE (MFILTER_LESS) implemented in PLUMED ${ }^{54}$ to create these two dynamic groups.

- Thereafter, we fixed the number of molecules constituting mother and daughter droplets. To do so, we used the RESTRAINT function implemented in PLUMED ${ }^{54}$ which can add harmonic and/or linear restaints on specific CVs. We applied the RESTRAINT function on two dynamic groups defined with the options LESS_THAN and MORE_THAN implemented in the ZDISTANCES function.

- We computed the number of contacts, $N_{C}$, between the two dynamic groups previously defined with MFILTER_MORE and MFILTER LESS using the function COORDINATTIONNUMBER implemented in PLUMED. ${ }^{54}$ This variable counts the number of contacts between two groups of atoms and is defined as $N_{\mathrm{C}}=\sum_{i} \sum_{j} s_{i j}$, where $s_{i j}=1$ if the contact between atoms $i$ and $j$ is formed and $s_{i j}=0$ otherwise. To make $N_{\mathrm{C}}$ differentiable, $s_{i j}$ is replaced with a switching function. We considered the RATIONAL switching function $s(r)=\frac{1-\left(\frac{r-D_{0}}{R_{0}}\right)^{n}}{1-\left(\frac{r-D_{0}}{R_{0}}\right)^{m}}$, where $n=6$ and $m=12$. We choose $D_{0}$ $=0.6375 \mathrm{~nm}$, as obtained from the position of the first peak of the radial distribution function calculated from the distances between the fluid molecules, which corresponds to the first coordination shell of the system. ${ }^{54}$ We then tuned the value of the parameter $R_{0}=0.8625 \mathrm{~nm}$ to allow the daughter droplet to separate completely from the mother droplet during fragmentation.

- Finally, we defined the continuous number of contacts as the mean value of the COORDINATIONNUMBER discussed above. To do so, we used the MEAN option implemented in the MULTICOLVAR module available in PLUMED. ${ }^{54}$

\section{ASSOCIATED CONTENT}

\section{S Supporting Information}

The Supporting Information is available free of charge on the ACS Publications website at DOI: 10.1021/acsnano.9b04454.

Numerical simulation methods and system characterization (PDF)

\section{AUTHOR INFORMATION}

\section{Corresponding Author}

*E-mail: francois.sicard@free.fr.

\section{ORCID 1}

François Sicard: 0000-0003-1408-7963

Jhoan Toro-Mendoza: 0000-0003-4916-7445

Alberto Striolo: 0000-0001-6542-8065

\section{Notes}

The authors declare no competing financial interest.

\section{ACKNOWLEDGMENTS}

The authors acknowledge V. Garbin and L. Botto for useful discussions. F.S. thanks M. Salvalaglio for fruitful discussion concerning the accelerated dynamics frameworks and P. Rousseau, A. Lesne, and M. Barbi for stimulating discussions regarding the biological implications of our findings. Via our membership of the UKs HEC Materials Chemistry Consortium, which is funded by EPSRC (EP/L000202), this work used the ARCHER UK National Supercomputing Service (http://www.archer.ac.uk). F.S. and A.S. acknowledge the support of the UK Engineering and Physical Sciences Research Council (EPSRC), under grant number 527889.

\section{REFERENCES}

(1) Leal-Calderon, F.; Poulin, P. Progress in Understanding Emulsion Metastability and Surface Forces. Curr. Opin. Colloid Interface Sci. 1999, 4, 223-230.

(2) Shui, L.; Eijkel, J.; van den Berg, A. Multiphase Flow in Microfluidic Systems - Control and Applications of Droplets and Interfaces. Adv. Colloid Interface Sci. 2007, 133, 35-49.

(3) Binks, B. Particles as Surfactants - Similarities and Differences. Curr. Opin. Colloid Interface Sci. 2002, 7, 21-41.

(4) Binks, B. P.; Desforges, A.; Duff, D. G. Synergistic Stabilization of Emulsions by a Mixture of Surface-Active Nanoparticles and Surfactant. Langmuir 2007, 23, 1098-1106.

(5) Pickering, S. Emulsions. J. Chem. Soc., Trans. 1907, 91, 20012021

(6) Qu, Y.; Huang, R.; Qi, W.; Qu, Q.; Su, R.; He, Z. Structural Insight into Stabilization of Pickering Emulsions with Fe3O4@SiO2 Nanoparticles for Enzyme Catalysis in Organic Media. Part. Part. Syst. Charact. 2017, 34, 1700117.

(7) Frelichowska, J.; Bolzinger, M.-A.; Valour, J.-P.; Mouaziz, H.; Pelletier, J.; Chevalier, Y. Pickering W/O Emulsions: Drug Release and Topical Delivery. Int. J. Pharm. 2009, 368, 7-15.

(8) Pan, M.; Kim, M.; Blauch, L.; Tang, S. Surface-Functionalizable Amphiphilic Nanoparticles for Pickering Emulsions with Designer FluidFluid Interfaces. RSC Adv. 2016, 6, 39926-39932. 
(9) Agrawal, M.; Gupta, S.; Stamm, M. Recent Developments in Fabrication and Applications of Colloid Based Composite Particles. J. Mater. Chem. 2011, 21, 615-627.

(10) Agrawal, G.; Agrawal, R. Janus Nanoparticles: Recent Advances in Their Interfacial and Biomedical Applications. ACS Appl. Nano Mater. 2019, 2, 1738-1757.

(11) Kirillova, A.; Marschelke, C.; Synytska, A. Hybrid Janus Particles: Challenges and Opportunities for the Design of Active Functional Interfaces and Surfaces. ACS Appl. Mater. Interfaces 2019, 11, 9643-9671.

(12) Miller, R.; Fainerman, V.; Kovalchuk, V.; Grigoriev, D.; Leser, M.; Michel, M. Composite Interfacial Layers Containing Micro-Size and Nano-Size Particles. Adv. Colloid Interface Sci. 2006, 128-130, $17-26$.

(13) Razavi, S.; Cao, K. D.; Lin, B.; Lee, K. Y. C.; Tu, R. S.; Kretzschmar, I. Collapse of Particle-Laden Interfaces under Compression: Buckling versus Particle Expulsion. Langmuir 2015, $31,7764-7775$.

(14) Sicard, F.; Striolo, A. Buckling in Armored Droplet. Nanoscale 2017, 9, 8567-8572.

(15) Bizmark, N.; Ioannidis, M. Nanoparticle-Stabilised Emulsions: Droplet Armouring vs. Droplet Bridging. Soft Matter 2018, 14, 64046408 .

(16) Luu, X.-C.; Yu, J.; Striolo, A. Nanoparticles Adsorbed at the Water/Oil Interface: Coverage and Composition Effects on Structure and Diffusion. Langmuir 2013, 29, 7221-7228.

(17) Ichii, T.; Suzuki, H.; Yomo, T. Micro-Droplet Model for Recursive Growth and Division Dynamics of the Cell. EPL 2011, 96, 48006.

(18) Caschera, F.; Rasmussen, S.; Hanczyc, M. An Oil Droplet Division-Fusion Cycle. ChemPlusChem 2013, 78, 52-54.

(19) Zwicker, D.; Seyboldt, R.; Weber, C.; Hyman, A.; Julicher, F. Growth and Division of Active Droplets Provides a Model for Protocells. Nat. Phys. 2017, 13, 408-413.

(20) Kotov, N. Inorganic Nanoparticles as Protein Mimics. Science 2010, 330, 188-189.

(21) Hanczyc, M. Droplets: Unconventional Protocell Model with Life-Like Dynamics and Room to Grow. Life 2014, 4, 1038-1049.

(22) Sikka, V.; Chattu, V. K.; Popli, R.; Galwankar, S.; Kelkar, D.; Sawicki, S.; Stawicki, S.; Papadimos, T. The Emergence of Zika Virus as a Global Health Security Threat: A Review and a Consensus Statement of the INDUSEM Joint working Group (JWG). J. Glob. Infect. Dis. 2016, 8, 3-15.

(23) Sevvana, M.; Long, F.; Miller, A.; Klose, T.; Buda, G.; Sun, L.; Kuhn, R.; Rossmann, M. Refinement and Analysis of the Mature Zika Virus Cryo-EM Structure at 3.1 A Resolution. Structure 2018, 26, $1169-1177$.

(24) Saey, T. H. Zika Gets the Most Extreme Close-up of Any Flavivirus. Sci. News 2018, 194, 15.

(25) Shekar, M. C.; Chary, B. B.; Srinivas, S.; Kumar, B. R.; Mahendrakar, M. D.; Varma, M. V. K. Effect of Ultrasonication on Stability of Oil in Water Emulsions. Int. J. Drug Delivery 2011, 3, 133140.

(26) Lad, V.; Murthy, Z. Enhancing the Stability of Oil-in-Water Emulsions Emulsified by Coconut Milk Protein with the Application of Acoustic Cavitation. Ind. Eng. Chem. Res. 2012, 51, 4222-4229.

(27) Huerre, A.; De Corato, M.; Garbin, V. Dynamic Capillary Assembly of Colloids at Interfaces with 10,000g Accelerations. Nat. Commun. 2018, 9, 3620.

(28) Mabille, C.; Leal-Calderon, F.; Bibette, J.; Schmitt, V. Monodisperse Fragmentation in Emulsions: Mechanisms and Kinetics. Europhys. Lett. 2003, 61, 708-714.

(29) Mulligan, M.; Rothstein, J. Deformation and Breakup of Microand Nanoparticle Stabilized Droplets in Microfluidic Extensional Flows. Langmuir 2011, 27, 9760-9768.

(30) Tcholakova, S.; Valkova, Z.; Cholakova, D.; Vinarov, Z.; Lesov, I.; Denkov, N.; Smoukov, S. Efficient Self-Emulsification via CoolingHeating Cycles. Nat. Commun. 2017, 8, 15012.
(31) Tolman, R. The Effect of Droplet Size on Surface Tension. J. Chem. Phys. 1949, 17, 333-337.

(32) Wu, N.; Lee, D.; Striolo, A. Anisotropic Particle Assemblies: Synthesis, Assembly, Modeling, and Applications; Elsevier: Amsterdam, 2018.

(33) Groot, R.; Warren, P. Dissipative Particle Dynamics: Bridging the Gap between Atomistic and Mesoscopic Simulation. J. Chem. Phys. 1997, 107, 4423-4435.

(34) Hamelberg, D.; Mongan, J.; McCammon, J. Accelerated Molecular Dynamics: A Promising and Efficient Simulation Method for Biomolecules. J. Chem. Phys. 2004, 120, 11919-11929.

(35) Binks, B.; Lumsdon, S. Influence of Particle Wettability on the Type and Stability of Surfactant-Free Emulsions. Langmuir 2000, 16, $8622-8631$

(36) Laio, A.; Parrinello, M. Escaping Free-Energy Minima. Proc. Natl. Acad. Sci. U. S. A. 2002, 99, 12562-12566.

(37) Kastner, J. Umbrella Sampling. Wiley Interdiscip. Rev.: Comput. Mol. Sci. 2011, 1, 932-942.

(38) Marchi, M.; Ballone, P. Adiabatic Bias Molecular Dynamics: A Method to Navigate the Conformational Space of Complex Molecular Systems. J. Chem. Phys. 1999, 110, 3697-3702.

(39) Sicard, F.; Striolo, A. Numerical Analysis of Pickering Emulsion Stability: Insights from ABMD Simulations. Faraday Discuss. 2016, 191, 287-304.

(40) de Gennes, P.-G.; Brochard-Wyart, F.; Quere, D. Capillarity and Wetting Phenomena: Drops, Bubbles, Pearls, Waves; SpringerVerlag: New York, 2004.

(41) Lei, Y.; Bykov, T.; Yoo, S.; Zeng, X. The Tolman Length: Is it Positive or Negative. J. Am. Chem. Soc. 2005, 127, 15346-15347.

(42) Fan, H.; Striolo, A. Nanoparticle Effects on the Water-Oil Interfacial Tension. Phys. Rev. E 2012, 86, 051610.

(43) Wi, H.; Cingarapu, S.; Klabunde, K.; Law, B. Nanoparticle Adsorption at Liquid-Vapor Surfaces: Influence of Nanoparticle Thermodynamics, Wettability, and Line Tension. Langmuir 2011, 27, 9979-9984.

(44) Bottone, M. G.; Santin, G.; Aredia, F.; Bernocchi, G.; Pellicciari, C.; Scovassi, A. Morphological Features of Organelles during Apoptosis: An Overview. Cells 2013, 2, 294-305.

(45) Aguilera-Gomez, A.; Rabouille, C. Membrane-Bound Organelles versus Membrane-Less Compartments and their Control of Anabolic Pathways in Drosophilia. Dev. Biol. 2017, 428, 310-317.

(46) Chen, J.; Stark, L. Crosstalk between NF- $\kappa$ B and Nucleoli in the Regulation of Cellular Homeostasis. Cells 2018, 7, 157.

(47) Elmore, S. Apoptosis: A Review of Programmed Cell Death. Toxicol. Pathol. 2007, 35, 495-516.

(48) Renehan, A.; Booth, C.; Potten, C. What is Apoptosis, and Why is it Important? BMJ. 2001, 322, 1536-1538.

(49) Caruso, S.; Poon, K. Apoptotic Cell-Derived Extracellular Vesicles: More Than Just Debris. Front. Immunol. 2018, 9, 1486.

(50) Wong, R. Apoptosis in Cancer: From Pathogenesis to Treatment. J. Exp. Clin. Cancer Res. 2011, 30, 87.

(51) Pak, T.; Archilha, N.; Mantovani, I.; Moreira, A.; Butler, I. The Dynamics of Nanoparticle-Enhanced Fluid Displacement in Porous Media-A Pore-Scale Study. Sci. Rep. 2018, 8, 11148.

(52) Gajewski, E.; Steckler, D.; Goldberg, R. Thermodynamics of the Hydrolysis of Adenosine 5'-Triphosphate to Adenosine 5'Diphosphate. J. Biol. Chem. 1986, 261, 12733-12737.

(53) Toyofuku, M.; Nomura, N.; Eberl, L. Types and Origins of Bacterial Membrane Vesicles. Nat. Rev. Microbiol. 2019, 17, $13-24$.

(54) Tribello, G.; Bonomi, M.; Branduardi, D.; Camilloni, C.; Bussi, G. PLUMED 2: New Feathers for an Old Bird. Comput. Phys. Commun. 2014, 185, 604-613. 